\title{
MICROSTRUCTURE AND MECHANICAL PROPERTIES OF NANOSTRUCTURED Ti-22Nb-10Zr COATINGS
}

\author{
${ }^{1,2}$ Emilio FRUTOS, ${ }^{3,4}$ Miroslav KARLÍK, ${ }^{5}$ José Antonio JIMÉNEZ, ${ }^{6}$ Tomáš POLCAR \\ ${ }^{1}$ Institute Pedro Nunes, Laboratory for Wear, Testing and Materials, Rua Pedro Nunes, 3030-199 Coimbra, \\ Portugal, EU, etorres@ipn.pt \\ ${ }^{2}$ CEMMPRE-Center for Mechanical Engineering, Materials and Processes, University of Coimbra, Rua Luís \\ Reis Santos, 3030-788, Coimbra, Portugal. \\ ${ }^{3}$ Department of Materials, Faculty of Nuclear Sciences and Physical Engineering, Czech Technical University \\ in Prague, Trojanova 13, 12000 Prague 2, Czech Republic, EU, Miroslav.Karlik@fffi.cvut.cz \\ ${ }^{4}$ Department of Physics of Materials, Faculty of Mathematics and Physics, Charles University, Ke Karlovu 5, \\ 12116 Prague 2, Czech Republic, EU \\ ${ }^{5}$ Centro Nacional de Investigaciones Metalúrgicas (CENIM-CSIC), Avd. Gregorio del Amo no 8, \\ 28040 Madrid, Spain, EU, jimenez@cenim.csic.es \\ ${ }^{6}$ Department of Control Engineering, Faculty of Electrical Engineering, Czech Technical University in Prague, \\ Technická 2, 16000 Prague 6, Czech Republic, EU, polcar@fel.cvut.cz
}

https://doi.org/10.37904/metal.2020.3540

\begin{abstract}
The design of implants and functional prostheses requires superficial modifications that promote fast and lasting osseointegration. Magnetron sputtering enables to design nanostructured and textured $\beta$-Ti rich Ti$22 \mathrm{Nb}-10 \mathrm{Zr}$ (wt.\%) coatings with variable mechanical properties (hardness and Young's modulus). Depending on the magnitude of the bias voltage used during deposition of the coating, martensitic transformation from the unstable $\beta$ (bcc) to $\alpha$ " (orthorhombic) phase is activated. This transformation induces compressive residual stresses modifying the tensile strength, hardness and Young's modulus. The residual stresses were measured by nanoindentation, the microstructure and phase evolution were characterized by $\mathrm{X}$-ray diffraction. The spatial phase distribution was determined by transmission electron microscopy. The calculated real hardness increases from 2.1 to $4.1 \mathrm{GPa}$ as the bias voltage is increased from 0 to $-148 \mathrm{~V}$. The calculus confirms that the coating has a non-linear elastic behavior.
\end{abstract}

Keywords: Biomaterials, Non-toxic beta-rich Ti coatings, Martensitic phase transformation, Residual stresses, Nano-mechanical characterization

\section{INTRODUCTION}

In recent years, considerable effort has been made in the development of implants and prostheses with a longer lifespan and better performance by surface modification techniques and/or by the design of coatings $[1,2]$. Nevertheless, the design of protective coatings, capable of interacting with a dysfunctional hard tissue, requires a balance of mechanical properties, such as high strength ( $\sim 800 \mathrm{MPa})$ and low Young's modulus, great fracture toughness and fatigue resistance, together with a high wear-corrosion resistance to body-fluids and bone bonding ability. In order to get this balance, the development of nanostructures for imparting multifunctionality should be included, since the bone/implant interface presents a better mechanical and biological compatibility when implant surface is composed of nanograins [3]. For this goal, magnetron sputtering presents clear advantages over other coating techniques by allowing a strict control of the homogeneity of thickness and chemical composition. 
The fabrication of nanostructured layers of metastable $\beta$-phase rich $\mathrm{Ti}$ alloys retained at room temperature requires the addition of $\beta$ phase stabilizing elements and the optimization of sputtering conditions. Young's modulus value may be adjusted as a function of the balance between different phases, since Young's modulus of the hexagonal $\alpha$ phase [4] is almost twice of the cubic $\beta$ phase $[5,6]$. Depending of the initial $\mathrm{Nb} / \mathrm{Zr}$ ratio, the increase of the bias voltage allows to induce a martensitic transformation $(\beta \rightarrow \alpha$ ") in situ during the sputtering deposition. In consequence it is possible to manipulate the mechanical properties as a result of higher or lower degree of $\beta \rightarrow \alpha$ " transformation. The main goal of this work is to characterize the magnitude of compressive residual stresses in $\mathrm{Ti}-22 \mathrm{Nb}-10 \mathrm{Zr}$ (wt.\%) coatings prepared by magnetron sputtering at bias voltage ranging from 0 to $-148 \mathrm{~V}$, and to evaluate their influence on hardness, yield and tensile strength.

\section{EXPERIMENTAL PROCEDURES}

Ti-22Nb-10Zr coatings have been deposited on (100) Si wafers using magnetron sputtering from $\mathrm{Ti}, \mathrm{Nb}$ and $\mathrm{Zr}$ targets (each of $99,99 \%$ purity). During the deposition process, the DC power was fixed at $371 \mathrm{~W}$ for Ti, at $34 \mathrm{~W}$ for $\mathrm{Nb}$ and at $20 \mathrm{~W}$ for $\mathrm{Zr}$; the bias voltage on the substrate has been modified and then kept constant from $0,-63,-98,-125$, to $-148 \mathrm{~V}$. Each deposition has been completed for about $2700 \mathrm{~s}$, resulting in a total film thickness of $\approx 600 \mathrm{~nm}$ (for more details see [7]). The phase evolution as a function of the bias voltage was determined by grazing incidence X-ray diffraction using Rigaku Smart Lab X-ray diffractometer equipped with a rotating $\mathrm{Cu}$ anode operating at $9 \mathrm{~kW}(45 \mathrm{kV}, 200 \mathrm{~mA})$ and a high-speed linear detector D/teX ultra 250. The XRD data refinement was performed using Rietveld analysis software TOPAS (Bruker AXS, version 4.2). The morphology and spatial distribution of the phases $\alpha$, $\alpha$ " and $\beta$ was characterized using a transmission electron microscope (TEM) JEOL 2200FS equipped with a NanoMegas ASTAR automated crystal orientation mapping system. Mechanical properties were measured by means of Micro Materials Nanotest-Vantage nanoindentor equipped with a Berkovich type indenter, using loads ranging from 1 to $10 \mathrm{mN}$, and loading and unloading strain rates of 0.05 and $0.2 \mathrm{~s}^{-1}$, respectively. A $15 \mathrm{~s}$ hold period was selected at the end of the loading phase. In all cases, mechanical properties values given for each load correspond to average values taken from at least 10 measurements.

\section{RESULTS AND DISCUSSION}

\subsection{Microstructure and phase composition of the coatings}

The coatings presented a mix of $\alpha-, \alpha$ "- and $\beta$-Ti phases. The relative amount (volume fraction) of these phases can be correlated with the bias voltage used for deposition, as shown in Figure 1a. This is directly related to the changes introduced in the adatom mobility when the bias voltage is increased [8]. In fact, if the mobility is in-
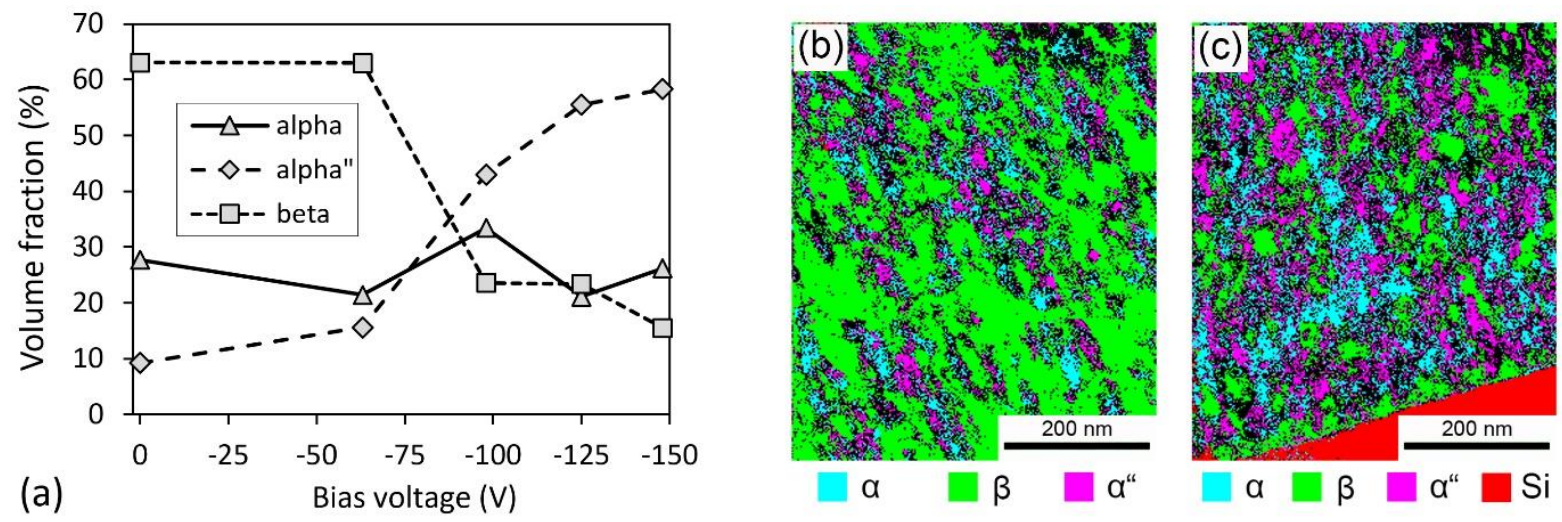

Figure 1 Evolution of the phase composition with negative bias voltage (a) and TEM nanodiffraction phase maps of Ti-22Nb-10Zr (wt.\%) coatings corresponding to bias voltage of $-63 \mathrm{~V}(\mathrm{~b})$, and $-148 \mathrm{~V}$ (c). 
sufficient, preferential orientations along lower-surface-diffusivity planes, i.e., in those planes with highsurface-energy, will grow and therefore the grain morphology of $\beta$ phase will be columnar type (Figure 1b). On the other hand, if this mobility is high enough (at high bias voltage values), surface diffusion among grains occurs and they start to grow towards the lowest-surface-energy plane. Thereby, texture and grain morphology of $\beta$ phase will change from columnar to equiaxial (Figure 1c).

\subsection{Mechanical properties}

\subsubsection{Real hardness calculation, $H_{0}$}

The presence of high compressive residual stresses in the coating has a direct impact in the loading-unloading indentation curve, and therefore in the measured hardness value $H$. In fact, the smaller the maximum indentation depth $h_{\max }$, the lower the plastic volume generated in the indentation and the smaller the mobility of statically stored dislocations (SSD) during the indentation are [9]. The combination of the low SSD mobility and the high geometrically necessary dislocations (GND) density generated in the small volume associated with shallow indentation, produces an increase in the real hardness $H_{0}$ of the coating (in the limit of infinite depth). The value $H_{0}$ can be determined from the Nix and Gao model [10]:

$\left(\frac{H}{H_{0}}\right)^{2}=1+h^{*} \cdot\left(\frac{1}{h_{\max }}\right)$

where $h^{*}$ is a characteristic depth, which is related to the indenter shape and the SSD density through $H_{0}$. However, deviations from this model have been observed in those cases where the grain size is included in the nano-scale and its size is comparable to the plastic volume generated in shallow indentations. Yang et al. [11] showed that the indentation size affects (typically at low indentation depths) experiments in an opposite manner, i.e., with increasing indentation depth the hardness increases, since the GNDs interact directly with grain boundaries which obstruct the movement of dislocations. Therefore, the plastic deformation can be confined in a single indented grain. A similar behavior is observed for the hardness values measured in our $\mathrm{Ti}-22 \mathrm{Nb}-10 \mathrm{Zr}$ coating once the substrate influence has been eliminated. Figure 2 shows atypical indentation size effect behavior, which is related to different plastic mechanism activated (twinning and slip systems) in the $\alpha, \alpha$ " and $\beta$ phases. In this figure it is possible to see how the $H^{2}$ versus $1 / h_{\max }$ curves for two bias voltage conditions of -63 and -125 Volts have three different trends when $1 / h_{\max }$ decreases. In the first part, $H^{2}$ values decrease until reaching a minimum value, A. Along this first part, hardness values depend mainly on the indentation size and the presence of compression residual stresses, which acts as a hardening element, and not on the different plastic mechanism activated on the different nano-grain sizes. From this minimum value, $H^{2}$ values increase (as has been shown by Yang et al. [11]) until reaching a maximum value, B. After this maximum, a decrease in the hardness values, $\mathbf{C}$, with increasing $h_{\max }$, is shown in Figure $\mathbf{2} \mathbf{b}$, which might indicate a change in the plastic mechanism because of a decrease in the dislocation density.
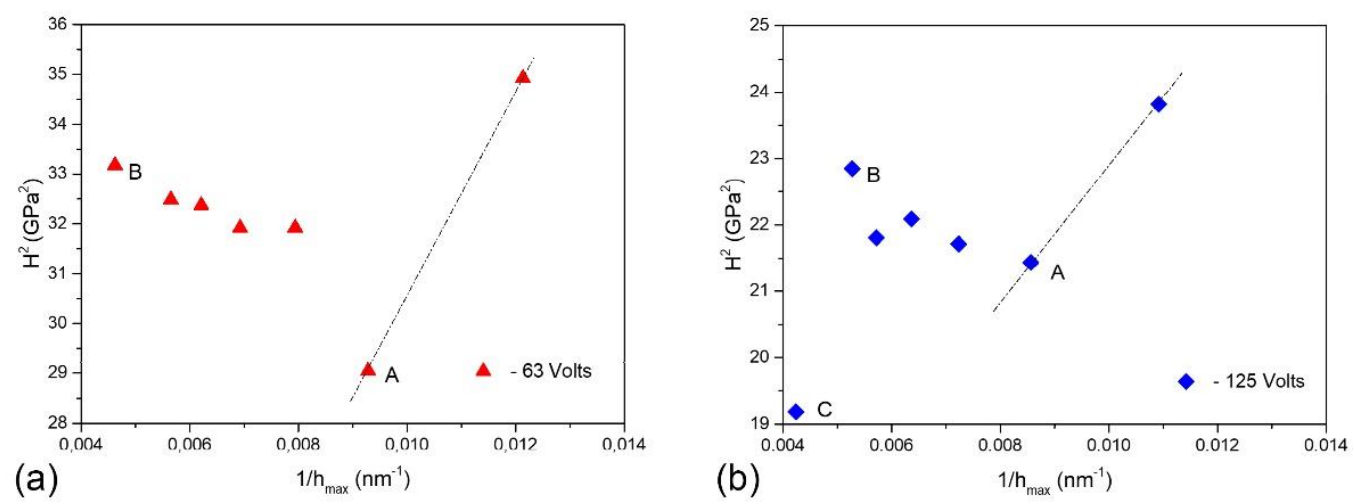

Figure 2 Atypical indentation size effect behavior, shown for two bias voltage conditions (-63 and $-125 \mathrm{~V})$, is related to different plastic mechanism activated (twinning and slip systems) in the $\alpha, \alpha$ " and $\beta$ phases. 
Table 1 Values corresponding to the real hardness values, $H_{0}$, the stress value $\sigma_{3}$ represents the value of $\sigma_{y}$ when $\rho$ is equal to 3 , the representative stress, $\sigma_{R e p}$, value, which is associated with the representative strain, $\varepsilon_{R e p}$, between 0.005 and 0.042 , the strain-hardening exponent, $n$, and finally, the stress values corresponding to a strain of $0.5 \%$ and $4.2 \%, \sigma_{0.005}$ and $\sigma_{0.042}$, respectively.

\begin{tabular}{|c|c|c|c|c|c|c|}
\hline $\begin{array}{c}\text { Bias voltage } \\
(\mathbf{V})\end{array}$ & $\begin{array}{c}\boldsymbol{H}_{0} \\
(\mathbf{G P a})\end{array}$ & $\begin{array}{c}\sigma_{3} \\
(\mathrm{GPa})\end{array}$ & $\begin{array}{c}\sigma \mathrm{\sigma Re} \\
(\mathrm{GPa})\end{array}$ & $\mathbf{n}$ & $\begin{array}{c}\sigma 0.005 \\
(\mathbf{M P a})\end{array}$ & $\begin{array}{c}\sigma 0.042 \\
(\mathbf{M P a})\end{array}$ \\
\hline 0 & 2.1 & 0.7 & 0.6 & 0.30 & 295 & 559 \\
\hline-63 & 3.2 & 1.1 & 1.1 & 0.67 & 235 & 980 \\
\hline-98 & 3.6 & 1.2 & 1.1 & 0.55 & 322 & 1050 \\
\hline-125 & 3.8 & 1.3 & 1.2 & 0.56 & 338 & 1108 \\
\hline-148 & 4.1 & 1.4 & 1.4 & 0.51 & 399 & 1192 \\
\hline
\end{tabular}

Nevertheless, by applying the Nix and Gao model to the hardness values that decrease with increasing maximum indentation depth, it is possible to get the real hardness values, $H_{0}$, as a function of the bias voltage increase. As can be seen in the Table 1, the $H_{0}$ value increases continuously from 2.1 to $4.1 \mathrm{GPa}$ when the bias voltage is increased from 0 to $-148 \mathrm{~V}$.

\subsubsection{Yield stress calculation, $\sigma_{y}$}

Ashby and Jones [12] and Tabor [13] formulated an empirical relationship, in which the hardness, $H_{0}$, represents the real hardness value (i.e. not the value obtained directly from the nano-indentation tests). The $H_{0}$ value is proportional to the yield strength, $\sigma_{y}$, at representative strain 0.08 through the equation:

$H_{0}=\rho \sigma_{y}$

where $\rho$ is a constant, which value is approximately equal to 3 for metals. In this way and using the $H_{0}$ values, obtained before, is possible to calculate the yield strength, $\sigma_{3},\left(\sigma_{3}\right.$ represents the value of $\sigma_{y}$ when $\rho$ is equal to 3) values as a function of the bias voltage increase (Table 1). As one might expect, this "yield strength" increases from 0.7 to $1.4 \mathrm{GPa}$ when the bias voltage is increased from 0 to $-148 \mathrm{~V}$. These high values prove that the presence of the hexagonal a phase, whose content is always between 21 and 33 wt.\% (Figure 1a, Figure 3a), has a clear hardening effect, hindering dislocation movement, because of a higher interaction number given by $\alpha / \alpha "$ interfaces.

\subsubsection{Compressive residual stress calculation, $\sigma_{r}$}

From the linear relationship, established by Sakharova et al [14], between $\left(P-P_{0}\right) / P_{0}$ ratio $(P$ corresponds to the maximum load reached in presence of residual stresses and $P_{0}$ without presence of residual stresses) and the normalized residual stress $\sigma_{r} / \sigma_{y}\left(\sigma_{r}\right.$ and $\sigma_{y}$ representing the residual stress and yield strength values, respectively), it is possible to get the residual stress magnitudes by:

$\frac{\left(P-P_{0}\right)}{P_{0}}=f\left(\varepsilon_{y}, n\right) \frac{\sigma_{r}}{\sigma_{y}}$

where $f\left(\varepsilon_{y}, n\right)$ varies depending on the nature of stresses and the other mechanical properties of the material (here, $\varepsilon_{r}=\sigma_{y} / \mathrm{E}$, and $n$ is strain-hardening exponent from the expression $\sigma=K \varepsilon^{n}$ - for more details see [15]). The function $f\left(\varepsilon_{y}, n\right)$ can be evaluated by fitting the linear coefficients of different materials and thus a particular function for compressive or tensile residual stresses can be obtained. 


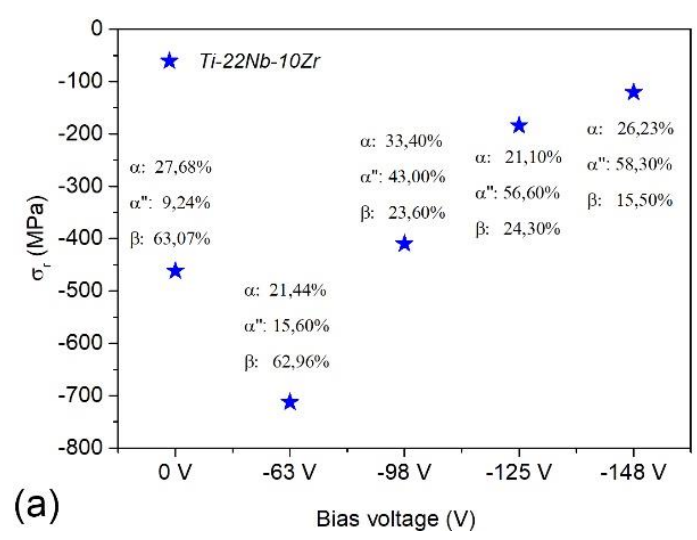

Figure 3 (a) Values of compressive residual stress, $\sigma_{r}$, as a function of bias voltage (including $\alpha$, $\alpha$ " and $\beta$ phase composition), (b) evolution of $\sigma_{r}$ with maximum indentation depth for bias voltage of -63 and $-148 \mathrm{~V}$.

Figure 3a shows the evolution of compressive residual stress values as a function of bias voltage increase. There is an important increase in the absolute value of the magnitude of compressive residual stresses from 460 to $-710 \mathrm{MPa}$ when the bias voltage is increased from 0 to $-63 \mathrm{~V}$. However, for the next bias voltages values, the magnitude of the compressive residual stresses shows, a priori, a contradictory evolution. In Figure $\mathbf{3 b}$ it is possible to see that for bias voltage values higher than $-63 \mathrm{~V}$, the magnitudes of compressive residual stresses are progressively decreasing until reaching $-120 \mathrm{MPa}$, corresponding to the sputtering deposition performed with a bias voltage of $-148 \mathrm{~V}$. This is a clear evidence that part of the energy transferred during sputtering deposition is used in the martensitic transformation from $\beta$ phase to $\alpha$ " phase, since the higher the bias voltage, the greater the $\alpha$ " content in the Ti-22Nb-10Zr coating. Consequently, this martensitic transformation causes a decrease in the magnitude of compressive residual stresses because the volume of the $\alpha$ " phase is lower than that of the $\beta$ phase [16]. This allows lattice relaxation and therefore a decrease in the magnitude of compressive residual stresses. Figure $\mathbf{3 b}$ shows the compressive residual stress evolution through the thickness coating, obtained from indentation tests with different maximum depths. In both cases (at -63 and $-148 \mathrm{~V}$ bias voltage) the stress magnitudes are almost constant through the thickness of the coatings. From these plots it can be concluded that the martensitic transformation takes place homogenously during the deposition process through the total thickness of the coating.

\section{CONCLUSIONS}

As the bias voltage is increased, the percentage and main textures of $\alpha$ (hcp), $\alpha$ " (orthorhombic) and $\beta$ (bcc) phases significantly change as a result of direct martensitic transformation ( $\beta \rightarrow \alpha$ "), which is activated thanks to the defects generated during sputtering deposition process through the total thickness of the Ti-22Nb-10Zr coating. The calculus of real hardness, $H_{0}$, shows how this value increase from 2.1 to $4.1 \mathrm{GPa}$ as the bias voltage is increased from 0 to $-148 \mathrm{~V}$. The simultaneous increase of $H_{0}$ and a" martensite content reveals the contribution of several factors, like grain size reduction, the increased defect amount generated during deposition and the increasing number of dislocation blocked in a greater number in $\alpha / \alpha$ " interface than $\alpha / \beta$ (semi-coherent) or $\alpha " / \beta$ (coherent) interfaces. The combination of all these factors can eliminate the classical softening effect shown in $\beta$-Ti alloys containing a high-volume fraction of $\alpha$ " phase. The calculus of strain hardening-exponent, $\mathrm{n}$, whose values are always higher than 0.5 for bias voltage between -63 and $-148 \mathrm{~V}$, confirms that $\mathrm{Ti}-22 \mathrm{Nb}-10 \mathrm{Zr}$ coating experience a non-linear elastic behavior, indicating that reversible martensitic transformation takes place once the external load is removed along unloading nanoindentation curve. On the other hand, the evolution of "tensile strength" $\left(\sigma_{0.042}\right)$ always shows values superior to $500 \mathrm{MPa}$ despite the increase of $\alpha$ " phase content with increase of the bias voltage. Therefore, the dislocations are pinned at $\alpha / \alpha$ " interfaces and, in combination with compressive residual stresses, promises high fracture toughness and fatigue resistance of the Ti-22Nb-10Zr coating regardless of the $\alpha$ " phase content. 
Finally, the decrease of the values of compressive residual stresses from -710 to $-120 \mathrm{MPa}$, when the bias voltage is increased from -63 to $-148 \mathrm{~V}$ shows an opposite trend than the $\alpha$ " martensite content. Since the volume of the $\alpha$ " phase is lower than that of the $\beta$ phase, lattice relaxation from the transformation results in a decrease in the compressive residual stresses magnitude.

\section{ACKNOWLEDGEMENTS}

\section{Financial support of the European Regional Development Fund (project No. CZ.02.1.01/0.0/0.0/15_003/0000485) is gratefully acknowledged.}

\section{REFERENCES}

[1] CHIEN, C.C., LIU, K.T., DUH, J.G., CHANG, K.W., CHUNG, K.H., Effect of nitride film coatings on cell compatibility, Dent. Mater., 2008, vol. 24, pp. 986-993.

[2] YANG, Y., Park, S., LIU, Y., LEE, K., KIM, H.S., KOH, J.T., MENG, X., KIM, K., Ji, H., WANG, X., ONG, J.L., Development of sputtered nanoscale titanium oxide coating on osseointegrated implant devices and their biological evaluation, Vacuum, 2008, vol. 83, pp. 569-574.

[3] ZAREIDOOST, A., YOUSEFPOUR, M., GHASEME, B., AMANZADEH, A., The relationship of surface roughness and cell response of chemical surface modification of titanium, J Mater. Sci. Mater. Med., 2012, vol. 23, no. 6, pp. 1479-1488.

[4] BOWEN, A.W., Omega Phase Embrittlement in Aged Ti15\% Mo, Scripta Metall. Mater., 1971, vol. 5, pp. 709-715.

[5] MATLAKHOVA, L.A., MATLAKHOVA, A.N., MONTEIRO, S.N., FEDOTOV, S:G., GOCHARENKO, B.A., Properties and structural characteristics of Ti-Nb-Al alloys, Mater. Sci. Eng. A, 2005, vol. 393, pp. 320-326.

[6] MOHAMED, A.H., HINOSHITA, K., MORINAGA, M., General approach to phase stability and elastic properties of $\beta$-type Ti-alloys using electronic parameters. Scripta Mater., 2006, vol. 55, pp. 477-480.

[7] FRUTOS, E., KARLÍK, M., POLCAR, T., The role of a" orthorhombic phase content on the tenacity and fracture toughness behavior of Ti-22Nb-10Zr coating used in the design of long-term medical implants, Appl. Surf. Sci. 2019, vol. 464, pp. 328-336.

[8] KAJIKAWA, Y., NODA, S., KOMIYAMA, H., Comprehensive perspective on the mechanism of preferred orientation in reative-sputter-deposited nitrides. J. Vacuum Sci. Technol. A, 2003, vol. 21, 1943.

[9] FRUTOS. E., MARTINEZ-MORILLAS, R., GONZALES-CARRASCO, J.L., Novel approaches to determining residual stresses by ultramicroindentation techniques: Application to sandblasted austenitic stainless steel. Acta Mater., 2010, vol. 58, pp 4191-4198.

[10] NIX, W.D., GAO, H.J., Indentation size effects in crystalline materials: a law for strain gradient plasticity. J. Mech. Phys. Solids., 1998, vol. 46, pp. 411-425.

[11] YANG, B., VEHOFF, H., Dependence of nanohardness upon indentation size and grain size-a local examination of the interaction between dislocations and grain boundaries. Acta Mater., 2007, vol. 55, pp. 849-856.

[12] ASHBY, M.F., JONES, D.R.H., Engineering Materials, Pergamon, Oxford, 1980

[13] TABOR, D., The hardness of metals. Clarendon Press, Oxford,1951.

[14] SAKHAROVA, N.A., PRATES, P.A., OLIVEIRA, M.C., FERNANDES, J.V., ANTUNES, J.M., A Simple Method for Estimation of Residual Stresses by Depth-Sensing Indentation. Strain, 2012, vol. 48, pp. 75-87.

[15] FRUTOS, E., KARLÍK, M., JIMENEZ, J.A., POLCAR, T., Contribution of non-linear elastic behaviour to the mechanical properties of nanostructured Ti-22Nb-10Zr coatings (in preparation).

[16] BÖNISCH, M., WAITZ, T., CALIN, M., SKROTZKI, W., ECKERT, J., Tailoring the Bain strain of martensitic transformations in Ti-Nb alloys by controlling the Nb content. Int. J. Plasticity, 2016, vol. 85, pp. 190-202. 